\title{
Medial Parietal Cortex Encodes Perceived Heading Direction in Humans
}

\author{
Oliver Baumann and Jason B. Mattingley \\ Queensland Brain Institute and School of Psychology, University of Queensland, St. Lucia, Queensland, 4072, Australia
}

The ability to encode and update representations of heading direction is crucial for successful navigation. In rats, head-direction cells located within the limbic system alter their firing rate in accordance with the animal's current heading. To date, however, the neural structures that underlie an allocentric or viewpoint-independent sense of direction in humans remain unknown. Here we used functional magnetic resonance imaging (fMRI) to measure neural adaptation to distinctive landmarks associated with one of four heading directions in a virtual environment. Our experiment consisted of two phases: a "learning phase," in which participants actively navigated the virtual maze; and a "test phase," in which participants viewed pairs of images from the maze while undergoing fMRI. We found that activity within the medial parietal cortex - specifically, Brodmann area 31-was modulated by learned heading, suggesting that this region contains neural populations involved in the encoding and retrieval of allocentric heading information in humans. These results are consistent with clinical case reports of patients with acquired lesions of medial posterior brain regions, who exhibit deficits in forming and recalling links between landmarks and directional information. Our findings also help to explain why navigation disturbances are commonly observed in patients with Alzheimer's disease, whose pathology typically includes the cortical region we have identified as being crucial for maintaining representations of heading direction.

\section{Introduction}

As humans navigate, they acquire knowledge about the layout of their environment. This information is encoded and stored in memory, allowing individuals to find their way back to a desired location. Navigation is thought to rely on the coordinated activity of several distinct neuronal populations. Specialized headdirection cells within the limbic system discharge as a function of the animal's current heading in the horizontal plane, independent of its location within the environment (Taube, 2007). These cells complement other neurons thought to underlie navigational behavior: "place cells" in the hippocampus (O'Keefe, 1976), which encode the animal's absolute location, and "grid cells" in the entorhinal cortex (Hafting et al., 2005), which discharge at multiple locations within an environment, in which these locations form a regular repeating pattern. Previous human studies have found evidence for place-like and grid-like representations (Ekstrom et al., 2003; Hassabis et al., 2009; Doeller et al., 2010), but to date no study has tested for representations relating exclusively to perceived heading.

Positional and directional spatial information can be encoded either egocentrically (i.e., relative to a person's location and orientation) or allocentrically (i.e., relative to other objects, independent of the location or orientation of the observer) (Klatzky,

\footnotetext{
Received June 16, 2010; revised July 16, 2010; accepted July 27, 2010.

This work was supported by an Australian Research Council and National Health and Medical Research Council Thinking Systems Grant. We gratefully acknowledge the Thinking Systems Team for their support and, in particular, Jack Valmadre for programming the virtual environment used in this study.

Correspondence should be addressed to Oliver Baumann, Queensland Brain Institute, The University of Queensland, St. Lucia, Queensland, 4072, Australia. E-mail: o.baumann@uq.edu.au. DOI:10.1523/JNEUROSCI.3077-10.2010

Copyright $\odot 2010$ the authors $\quad 0270-6474 / 10 / 3012897-05 \$ 15.00 / 0$
}

1998; Burgess, 2006). Spiers and Maguire (2007) investigated how humans encode egocentric direction and distance from a single target location during an active navigation task within a virtual environment. They found that activity in the medial prefrontal cortex was modulated by distance from the target and that activity in the posterior parietal cortex was positively correlated with egocentric direction with respect to the location of the goal. To date, however, no study in humans has obtained direct evidence for a neural representation of allocentric heading.

In humans, the retrosplenial complex (RSC) and precuneus are likely to play an important role in perceived heading. Acquired lesions of these posterior medial brain regions can cause severe impairments in forming and recalling links between landmark identity and directional information (Takahashi et al., 1997), a condition referred to as "heading disorientation" (Aguirre and D’Esposito, 1999). Although suggestive, however, such clinical case reports typically involve patients with large and often poorly defined lesions and so do not permit precise localization of function.

The goal of our study was to identify brain regions that underlie the representation of allocentric heading direction. We developed a virtual navigation task to identify neuronal populations that change their activity pattern depending on perceived heading. Our experiment consisted of two phases: a "learning phase," in which participants actively navigated the virtual maze; and a "test phase," in which participants viewed pairs of images from the maze while undergoing high-field functional magnetic resonance imaging (fMRI). We used fMRI adaptation (Krekelberg et al., 2006) (see Materials and Methods) to determine whether a distinct and localized neural representation is selective for heading direction in humans. Activity within any such region should be sig- 
nificantly attenuated for trials in which pairs of images represent the same heading direction (e.g., north-north) relative to trials in which pairs of images represent different headings (e.g., west-north).

\section{Materials and Methods}

Participants. Eighteen healthy, adult volunteers gave their informed consent to participate in the study, which was approved by the Human Research Ethics Committee of the University of Queensland. Data from five participants were excluded from the analysis because their behavioral performance during the test phase was below our a priori criterion of $90 \%$ correct responses (as outlined below). The remaining 13 participants (six females) ranged from 18 to 39 years of age (mean, 26 years); all were right-handed.

Behavioral training and fMRI study. To test for neural responses associated with perceived heading, we developed a navigation task in which participants first familiarized themselves with the layout of a virtual maze (the learning phase). They then viewed pairs of landmarks representing either the same heading direction (e.g., north-north) or different heading directions (e.g., west-north). Our aim was to identify neuronal populations that change their activity pattern depending on perceived heading. To detect these differential activation patterns, we used fMRI adaptation, an approach that can reveal the functional properties of subpopulations of neurons within an imaging voxel (Krekelberg et al., 2006). The technique relies on the observation that responses of neurons tuned to a given stimulus (the "probe") are reduced when it is preceded by an identical or related stimulus (the "prime") compared with conditions in which the probe and prime stimuli are unrelated (Grill-Spector et al., 2006).

Learning phase. In the learning phase of our study, participants completed five, 1-h training sessions, which consisted of a series of search tasks that required them to navigate as directly and quickly as possible, using a joystick held in their right hand, to various landmarks positioned at the periphery of a virtual maze (Fig. 1a). The maze was a Cartesian grid, made up of 10 corridors (five north-south corridors and five eastwest corridors). The two ends of each corridor contained a landmark in the form of a distinct symbol. The positions of the landmarks $(n=$ 20) within the maze were randomized across participants, to avoid any confounding effects that might arise as a consequence of particular groupings of symbols.

The participants' task was to navigate as quickly and directly as possible to the location of one of the 20 landmarks, indicated by a small cue (an image of the landmark) at the top of the computer screen. On every trial, participants started from the center of the maze (Fig. 1a, blue spot) and faced "north." At the end of each trial, the efficiency of the path taken by the participant was measured, in terms of both the time required to navigate to the cued location and the distance traversed. This information was flashed to participants after each trial to motivate them to improve their performance and thus to become more familiar with the spatial layout of the virtual maze. In each of the five training sessions, participants completed three to six blocks of 20 trials each. In every block of trials, the participants had to navigate to each of the 20 symbols in random order. We used the Blender open-source three-dimensional content creation suite (The Blender Foundation) to create the virtual maze and to administer the learning phase. Within the maze, each corridor had a (virtual) length of $30 \mathrm{~m}$, a height of $3.5 \mathrm{~m}$, and a width of $2.5 \mathrm{~m}$. The wall at the end of each corridor contained a landmark in the b

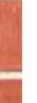
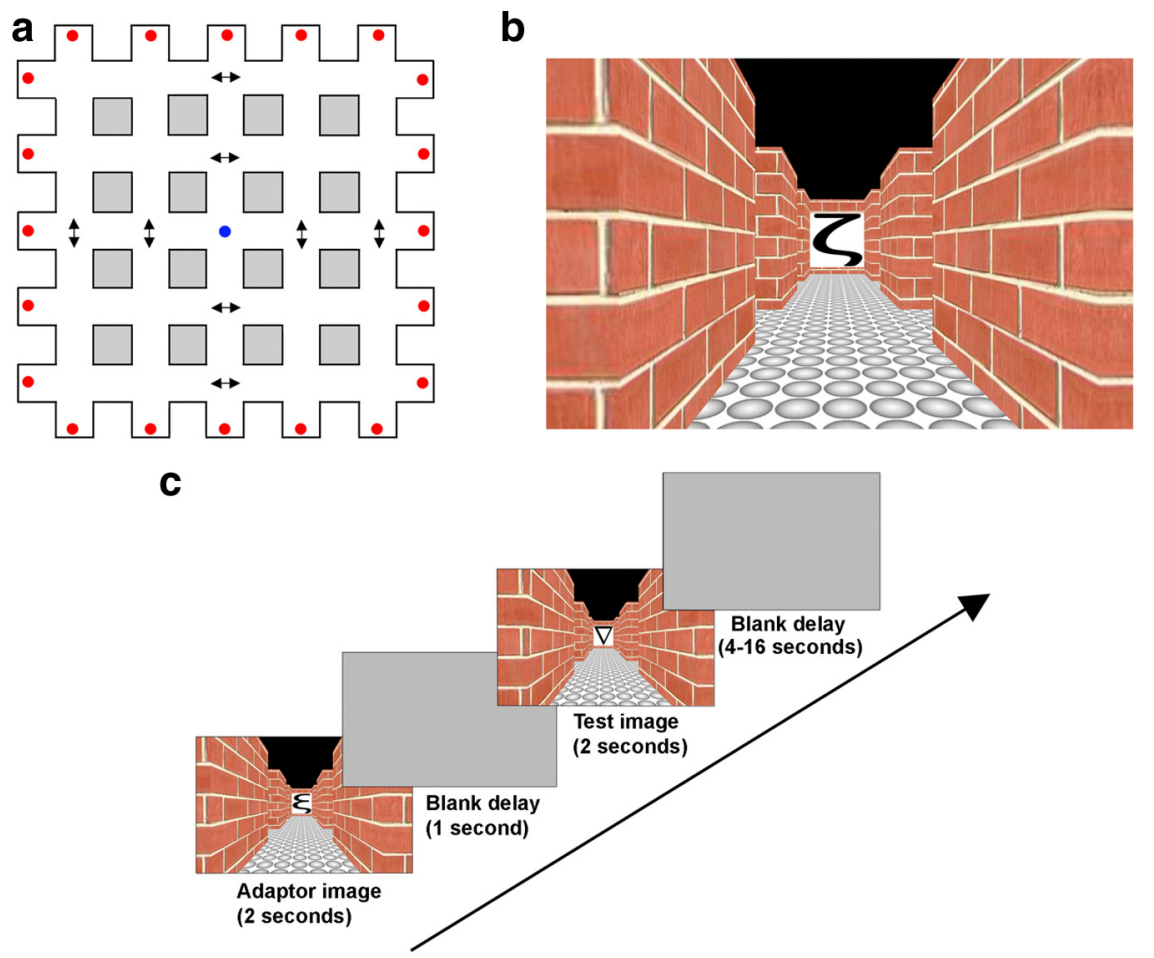

b . :

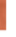

Figure 1. Schematics of the virtual environment and spatial judgment task used to examine the representation of allocentric heading. $\boldsymbol{a}$, Aerial perspective of the virtual maze (never seen by participants) used in the learning phase. The red dots indicate the lhe 20 symbols that acted as landmarks; the single blue spot represents the center of the virtual maze. The arrows of images depicting landmarks representing the same heading direction (repeated trials) or different heading directions (novel trials). Note that participants never viewed the same landmark symbol twice within a trial pair.

form of a black symbol on a white background. The symbols were letters from the Tagalog and Greek alphabets, as well as astronomical signs. The maze environment was constructed so that participants were never able to see more than one landmark at a time as they moved along the corridors (Fig. 1a). This ensured that the relationship between the positions of the landmarks had to be encoded entirely on the basis of an internal, allocentric representation of the layout of the virtual maze.

Test phase. In the test phase, we used fMRI to measure neural responses as participants viewed pairs of static, snapshot images of landmarks they had seen previously within the virtual maze during the learning phase (Fig. 1b). They were instructed to press one of two buttons using their right thumb to indicate whether the position represented by the static image of the landmark was on the left or right of the center point of the maze (Fig. 1a, blue spot). Note that, although the purpose of the behavioral task was to prompt participants to recall the spatial layout of the maze, the judgment they were required to make was independent of heading direction.

Each trial of the fMRI task consisted of a pair of landmark images, comprising the initial adapter image for $2 \mathrm{~s}$, followed by a blank screen for $1 \mathrm{~s}$, and then a test image for $2 \mathrm{~s}$ (Fig. 1c). Crucially, each image pair involved either a "novel" heading (in which adapter and test images came from the ends of corridors with different headings, e.g., west-north) or a "repeated" heading (in which adapter and test images came from the ends of corridors with the same heading, e.g., north-north). To avoid any neural adaptation to repetition of identical symbols, participants were never shown the same symbols in a repeated heading trial. Also, because participants were never required to make explicit judgments on whether successive symbols within a trial represented the same or a different heading, we avoided any potential contribution from repetition of simple motor responses. As an additional control, symbol pairs displayed in the novel and repeated trials were matched with respect to their distance from the central reference point and from one another, to avoid any potential contribution from representations of learned place information. 
Before the fMRI experiment, participants completed 20 training trials to familiarize themselves with the task. In the fMRI experiment, participants completed four scanning runs, for a total of 80 trials in each of the repeated and novel conditions. The sequencing of the trials, and the temporal jittering of rest periods, was optimized with optseq 2 software (http://surfer.nmr.mgh.harvard.edu/optseq/).

MRI acquisition and data analysis. Whole-brain echo-planar imaging (EPI) was conducted using a 4 T Bruker MedSpec MRI scanner (Bruker Biosciences) with a transverse electromagnetic head coil. For the EPI, 37 axial slices were acquired in an interleaved order, using a gradient echo echo-planar $\mathrm{T} 22^{\star}$-sensitive sequence (repetition time, $2200 \mathrm{~ms}$; echo time, $30 \mathrm{~ms}$; flip angle, $90^{\circ}$; matrix, $64 \times 64$; field of view, $192 \times 192 \mathrm{~mm}$; voxel size, $3 \times 3 \times 3 \mathrm{~mm}$. In addition to the EPI, we also acquired a field map (same resolution/slices as the EPI; repetition time, $900 \mathrm{~ms}$; echo time 1, $5.4 \mathrm{~ms}$; echo time 2, $10.8 \mathrm{~ms}$ ) and a T1-weighted structural $1 \mathrm{~mm}$ cubic inversion recovery-prepared fast low-angle shot sequence threedimensional scan. A liquid crystal display projector $(1024 \times 768$ resolution) backprojected the visual stimuli onto a screen positioned at the end of the scanner bed. Participants lay on their backs within the bore of the magnet and viewed the stimuli comfortably via a $45^{\circ}$ angled mirror that reflected the images displayed on the screen. The distance to the screen was $265 \mathrm{~cm}$ ( $15 \mathrm{~cm}$ from eyes to mirror), and the visible part of the screen encompassed $\sim 21^{\circ} \times 11^{\circ}$ of visual angle $(98 \times 50 \mathrm{~cm})$. To minimize head movement, all participants were stabilized with tightly packed foam padding surrounding the head.

Image processing and statistical analysis of fMRI data were performed using SPM5 (Wellcome Department of Imaging Neuroscience, University College London, London, UK). Functional data volumes were slicetime corrected, realigned to the first volume and unwarped using individual field maps. A T2*-weighted mean image of the unsmoothed images was coregistered with the corresponding anatomical T1-weighted image from the same individual. The individual $\mathrm{T} 1$ image was used to derive the transformation parameters for the stereotaxic space using the SPM5 template [Montreal Neurological Institute (MNI) template], which was then applied to the individual coregistered EPI images. The voxel sizes of the normalized images were $2 \mathrm{~mm}^{3}$. Images were then smoothed with an $8 \mathrm{~mm}$ full-width half-maximum isotropic Gaussian kernel. At the single-participant level, we applied a high-pass filter (cutoff, $128 \mathrm{~s}$ ) to remove baseline drifts. The adapter and test stimuli (novel or repeated) were modeled separately as $2 \mathrm{~s}$ events convolved with a hemodynamic response function, with predicted responses always covering the entire period. Our principal comparison involved the effect of repeated headings relative to novel headings. For each participant, statistical parametric maps of the $t$ statistic were generated from linear contrasts for the condition of novel $>$ repeated. These contrasts of parameter estimates were then included in a second-level group analysis using single-sample $t$ tests on the contrast images obtained from each participant. The time course of the average percentage blood oxygenation leveldependent (BOLD) signal-change was extracted using MarsBaR (http://marsbar.sourceforge.net/).

\section{Results}

\section{Learning phase}

As participants learned the layout of the virtual maze across the five training sessions, we recorded the spatial and temporal efficiency of the paths they took to reach the cued landmark. "Spatial efficiency" was defined as the ratio of the minimum distance between the starting point and the target landmark, and the actual length of the path taken by the participant. "Temporal efficiency" was defined as the ratio of the minimum amount of time necessary to travel between the starting point and the location of the target landmark, and the time actually taken by the participant to reach the target landmark. In the final training session, participants obtained an average \pm SE spatial efficiency of $99.15 \pm 0.28 \%$ and an average \pm SE temporal efficiency of $98.79 \pm 0.40 \%$. These figures indicate that participants successfully reached a ceiling of performance in learning the layout of the

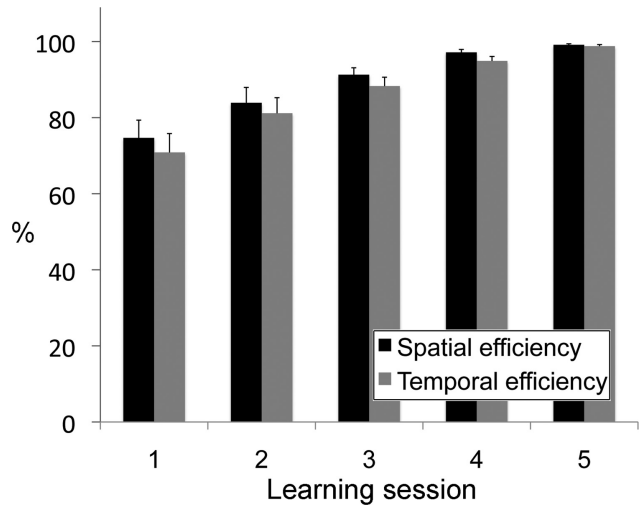

Figure 2. Mean values, expressed as a percentage of optimal efficiency, for spatial and temporal behavioral measures ( $\pm 1 \mathrm{SE}$ ) across the five learning sessions.

virtual maze, a crucial prerequisite for our goal of identifying the neural substrates of perceived heading in the test phase of the study. The behavioral results for all five learning sessions are displayed in Figure 2.

\section{Test phase}

Statistical analyses of the behavioral data acquired during the test phase revealed no difference between the time required by participants to respond in the repeated condition (mean $\pm \mathrm{SE}$, $1031 \pm 66 \mathrm{~ms}$ ) and the novel condition (mean $\pm \mathrm{SE}, 1050 \pm 73$ $\mathrm{ms}$; two-tailed paired $t$ test, $t_{(12)}=-0.88, p=0.39$ ). Moreover, there was no difference between accuracy measures for the two conditions (repeated condition, mean $\pm \mathrm{SE}, 99.03 \pm 0.32 \%$ vs novel condition, mean $\pm \mathrm{SE}, 98.94 \pm 0.31 \%$; two-tailed paired $t$ test, $\left.t_{(12)}=-0.37, p=0.72\right)$. These findings confirm that the novel and repeated trials of the behavioral task were well matched in terms of their level of difficulty.

For the fMRI analyses, statistical parametric maps of the $t$ statistic were generated from linear contrasts for the condition of novel $>$ repeated, separately for each participant. These contrasts of parameter estimates were then included in a second-level group analysis using single-sample $t$ tests on the contrast images obtained from each participant. This resulted in whole-brain, random effects (for the comparison of novel $>$ repeated and repeated $>$ novel). The results of the random-effects analysis of activity across the whole brain for the contrast (novel $>$ repeated) revealed a single, statistically significant activation cluster (onetailed paired $t$ test, $t_{(12)}=10.05, p=0.027$, familywise error corrected for multiple comparisons on voxel-level, cluster size of 4 ), within the precuneus of the left medial parietal lobe [Brodmann area (BA) 31; MNI coordinates of the peak voxel: -14 , $-66,20]$ (Fig. $3 a-c$ ). This region exhibited a reduced BOLD response for trial pairs in which the two images represented the same heading direction (indicating neural adaptation) compared with trial pairs in which the images represented different headings (Fig. $3 d$ ). The contrast (repeated $>$ novel) did not yield any significant activity.

To check for the existence for any subthreshold activation within the right medial parietal region (i.e., the area homologous to the left hemisphere site within which we found a significant adaptation effect in the whole-brain analysis), we conducted a separate region of interest analysis using MarsBaR. The region of interest was defined as a sphere (5 mm radius) with its center located at MNI coordinates of $14,-66,20$. A paired $t$ test revealed a marginally significant trend for an adaptation effect (i.e., a 
greater response for novel versus repeated conditions) within the right medial parietal region $\left(t_{(12)}=2.01, p=0.068\right)$. To check for the existence of any subthreshold activations associated with our manipulation of allocentric heading in other brain areas, we conducted an additional analysis of the fMRI data using a more liberal, uncorrected threshold of $p=$ 0.001 . This analysis revealed a small region of increased activity in the novel versus repeated condition that was restricted to the dorsal striatum bilaterally (MNI coordinates of the peak voxel: 24, 12, 2 and $-20,0$, 4). This region has been implicated previously in the retrieval of spatial information during active navigation (Iaria et al., 2003; Doeller et al., 2008; Baumann et al., 2010) but to date has not been associated explicitly with perceived heading.

\section{Discussion}

The goal of the present study was to identify brain structures involved in representing allocentric heading direction within a learned environment. We used fMRI to measure neural adaptation to pairs of images representing distinctive landmarks from the ends of corridors within a virtual maze. After an initial learning phase conducted over several days outside the scanner, participants viewed pairs of landmark images from the same heading (repeated trials) or from different headings (novel trials). We predicted that brain areas that encode or maintain allocentric heading information should show attenuated responses, consistent with repetition suppression (Grill-Spector et al., 2006; Krekelberg et al., 2006), in repeated versus novel trials.

Analysis of the fMRI data revealed a single brain region located in the medial parietal cortex (BA 31), in which activity was modulated by learned heading. The activation cluster was located within the so-called RSC, a region that has been functionally defined by Epstein (2008) on the basis of its activation during navigational tasks and during passive viewing of navigationally relevant stimuli. The RSC is close to, but distinct from, the anatomically defined retrosplenial cortex (encompassing only BA 29 and BA 30) (Vann et al., 2009) and extends superiorly into the posterior cingulate and posteriorly into the parietal-occipital sulcus/anterior calcarine region (Brodmann areas 23 and 31). We also observed a small cluster of reduced activity for repeated versus novel trials in the bilateral dorsal striatum, but this response was well below the threshold for significance and is thus not considered further here. Although the activation we observed within the RSC clearly indicates that this cortical region is involved in representing allocentric heading, the underlying mechanism of the suppression effect is yet to be determined. One possibility is that, in novel (i.e., different heading) trials, two distinct representations of allocentric orientation must be retrieved. In contrast, in repeated (i.e., same heading) trials, it is not necessary to retrieve orientation information for the second stimulus of the image pair, because this representation would already be active within relevant RSC neurons.
In previous $\mathrm{AMRI}$ studies, the RSC has been found to be strongly active during scene viewing, scene imagery, and scene memory (Epstein and Kanwisher, 1998; Epstein et al., 2007). Activity in the RSC during active navigation in a virtual environment has also been found to correlate with behavioral measures of map expertise (Wolbers and Büchel, 2005). More recently, Doeller et al. (2010) used fMRI to reveal grid-like representations in human entorhinal cortex. These investigators also observed activity in medial parietal areas associated with heading direction, but their task and display configurations did not permit disambiguation of activity related to allocentric heading per se, from activity evoked by visually identical landmarks associated with common headings within the virtual environment. In our task, participants were never shown the same landmark in repeated (or novel) trials, thus ensuring that the adaptation effect we observed for allocentric heading within RSC cannot be attributed to visual repetition of identical landmarks across experimental conditions.

The human ability to perceive and encode allocentric heading is consistent with the existence of so-called head-direction cells in the rodent limbic system (Taube, 2007). Head-direction cells alter their rate of firing in accordance with the animal's directional heading, so that, for example, a particular neuron might discharge whenever the animal points its head "north," independent of its location in the environment. It remains unknown whether rodent parietal cortex contains neural populations sensitive to perceived heading or whether the rodent head-direction system 
shares functional properties with the human system for representing allocentric heading. This will be an important line of investigation for future studies.

The nature of the representations underlying human spatial cognition has long been the subject of intense debate (Burgess, 2006). An influential review by Wang and Spelke (2002) suggested that spatial memory might be solely supported by egocentric or viewpoint-dependent representations. In contrast, our findings provide clear support for the existence of a viewpointindependent representation of spatial relationships. Previous investigations have shown that navigationally important landmarks are encoded incidentally (and possibly without explicit awareness) within the parahippocampal region during learning of the layout of a virtual environment (Janzen and van Turennout, 2004). Our findings extend these earlier observations by demonstrating that, in human RSC, important landmarks are encoded in a manner that reflects the allocentric directions in which they were observed during learning.

More broadly, our findings may have important applications beyond the laboratory. It should be possible, for example, to develop more refined behavioral probes of topographical disorientation in patients with acquired lesions of medial parietal cortex, by incorporating measures of allocentric heading perception (Takahashi et al., 1997; Aguirre and D’Esposito, 1999). In addition, in patients with Alzheimer's disease it has been shown that atrophy and hypometabolism in the medial parietal lobe correlate with the onset and severity of memory loss and that these changes typically precede the widespread cognitive decline observed later in the disease (Scahill et al., 2002; Nestor et al., 2003). Our finding that the medial parietal cortex normally plays a key role in representing allocentric heading thus also sheds new light on why patients with Alzheimer's disease frequently develop topographical disorientation as an early sign of disease progression (Monacelli et al., 2003).

\section{References}

Aguirre GK, D'Esposito M (1999) Topographic disorientation: a synthesis and taxonomy. Brain 122:1613-1628.

Baumann O, Chan E, Mattingley JB (2010) Dissociable neural circuits for encoding and retrieval of object locations during active navigation in humans. Neuroimage 49:2816-2825.

Burgess N (2006) Spatial memory: how egocentric and allocentric combine. Trends Cogn Sci 10:551-557.

Doeller CF, King JA, Burgess N (2008) Parallel striatal and hippocampal systems for landmarks and boundaries in spatial memory. Proc Natl Acad Sci U S A 105:5915-5920.

Doeller CF, Barry C, Burgess N (2010) Evidence for grid cells in a human memory network. Nature 463:657-661.
Ekstrom AD, Kahana MJ, Caplan JB, Fields TA, Isham EA, Newman EL, Fried I (2003) Cellular networks underlying human spatial navigation. Nature 425:184-188.

Epstein RA (2008) Parahippocampal and retrosplenial contributions to human spatial navigation. Trends Cogn Sci 12:388-396.

Epstein R, Kanwisher N (1998) A cortical representation of the local visual environment. Nature 392:598-601.

Epstein RA, Parker WE, Feiler AM (2007) Where am I now? Distinct roles for parahippocampal and retrosplenial cortices in place recognition. J Neurosci 27:6141-6149.

Grill-Spector K, Henson R, Martin A (2006) Repetition and the brain: neural models of stimulus-specific effects. Trends Cogn Sci 10:14-23.

Hafting T, Fyhn M, Molden S, Moser MB, Moser EI (2005) Microstructure of a spatial map in the entorhinal cortex. Nature 436:801-806.

Hassabis D, Chu C, Rees G, Weiskopf N, Molyneux PD, Maguire EA (2009) Decoding neuronal ensembles in the human hippocampus. Curr Biol 19:546-554.

Iaria G, Petrides M, Dagher A, Pike B, Bohbot VD (2003) Cognitive strategies dependent on the hippocampus and caudate nucleus in human navigation: variability and change with practice. J Neurosci 23:5945-5952.

Janzen G, van Turennout M (2004) Selective neural representation of objects relevant for navigation. Nat Neurosci 7:673-677.

Klatzky RL (1998) Allocentric and egocentric spatial representations: definitions, distinctions, and interconnections. In: Spatial cognition. An interdisciplinary approach to representing and processing spatial knowledge (Freska C, Havbel C, eds), pp 1-17. Heidelberg: Springer.

Krekelberg B, Boynton GM, van Wezel RJ (2006) Adaptation: from single cells to BOLD signals. Trends Neurosci 29:250-256.

Monacelli AM, Cushman LA, Kavcic V, Duffy CJ (2003) Spatial disorientation in Alzheimer's disease: the remembrance of things passed. Neurology 61:1491-1497.

Nestor PJ, Fryer TD, Smielewski P, Hodges JR (2003) Limbic hypometabolism in Alzheimer's disease and mild cognitive impairment. Ann Neurol 54:343-351.

O'Keefe J (1976) Place units in the hippocampus of the freely moving rat. Exp Neurol 51:78-109.

Scahill RI, Schott JM, Stevens JM, Rossor MN, Fox NC (2002) Mapping the evolution of regional atrophy in Alzheimer's disease: unbiased analysis of fluid-registered serial MRI. Proc Natl Acad Sci U S A 99:4703-4707.

Spiers HJ, Maguire EA (2007) A navigational guidance system in the human brain. Hippocampus 17:618-626.

Takahashi N, Kawamura M, Shiota J, Kasahata N, Hirayama K (1997) Pure topographic disorientation due to right retrosplenial lesion. Neurology 49:464-469.

Taube JS (2007) The head direction signal: origins and sensory-motor integration. Annu Rev Neurosci 30:181-207.

Vann SD, Aggleton JP, Maguire EA (2009) What does the retrosplenial cortex do? Nat Rev Neurosci 10:792-802.

Wang R, Spelke E (2002) Human spatial representation: insights from animals. Trends Cogn Sci 6:376.

Wolbers T, Büchel C (2005) Dissociable retrosplenial and hippocampal contributions to successful formation of survey representations. J Neurosci 25:3333-3340. 